\title{
Acknowledgment to Reviewers of Marine Drugs in 2021
}

\section{Marine Drugs Editorial Office}

MDPI AG, St. Alban-Anlage 66, 4052 Basel, Switzerland

Rigorous peer-reviews are the basis of high-quality academic publishing. Thanks to the great efforts of our reviewers, Marine Drugs was able to maintain its standards for the high quality of its published papers. Thanks to the contribution of our reviewers, in 2021, the median time to first decision was 12 days and the median time to publication was 29 days. The editors would like to extend their gratitude and recognition to the following reviewers for their precious time and dedication, regardless of whether the papers they reviewed were finally published:

Abdelfattah, Mohamed

Abdel-Latif, Hany M. R.

Abdollahi, Mehdi

Acquaviva, Rosaria

Adachi, Kohsuke

Adamczyk-Grochala, Jagoda

Adhikary, Till

Agòcs, Attila

Ahmed, Atallah F.

Aigle, Bertrand

Airs, Ruth Louise

Akiyama, Masahiro

Alarcón López, Francisco Javier

Albano, Francesco

Albericio, Fernando

Albini, Adriana

Aleu, Josefina

Allen, Scott

Alonso, Juan Rubio

Altemimi, Ammar

Alvarez, Ana I.

Álvarez, Enrique Domínguez

Alvariño, Rebeca

Aminin, Dmitry

Amoutzias, Grigoris

Amsler, Charles

Ana, Borrego-Sánchez

Anastyuk, Stanislav D.

Andó, István

Andrianasolo, Eric

Angelopoulou, Athina

Anniballi, Fabrizio

Antunes, Joana

Antunes, Jorge

Apicella, Antonio
Aprotosoaie, Ana Clara

Arai, Masayoshi

Arakawa, Osamu

Arellano, Juan B.

Arisaka, Yoshinori

Arita, Masanori

Arroba, Ana I.

Arteaga, Jesús Fernández

Artyukhin, Alex

Asamizu, Shumpei

Ashworth, Matt P.

Atobe, Masakazu

Avila-Román, Javier

Avilés, Francesc Xavier

Avni, Dorit

Ayala, Juan

Azam, Layla

Bacchiocchi, Simone

Bae, Ji-Yeong

Bae, Tae Soo

Baek, Kyung In

Baharum, Syarul Nataqain

Baindara, Piyush

Baker, Mark D.

Baker-Austin, Craig

Balen, Biljana

Baltz, Richard

Banskota, Arjun H.

Barbaric, Monika

Bardají, Eduard

Barh, Debmalya

Barnathan, Gilles

Barraja, Paola

Bartosz, Grzegorz

Basile, Livia 
Batista, Irineu

Beedessee, Girish

Behringer, Erik

Beld, Joris

Bello, Claudia

Belluzzi, Elisa

Belosludtsev, Konstantin

Ben-Gigirey, Begoña

Beppu, Fumiaki

Bergandi, Loredana

Berger, Stefan

Berillis, Panagiotis

Berrue, Fabrice

Bertrand, Samuel

Bezirtzoglou, Eugenia

Bharath, Leena P.

Biagini, Giuseppe

Biernasiuk, Anna

Bilancio, Antonio

Binda, Elisa

Biré, Ronel

Birsa, Mihail Lucian

Bissoyi, Akalabya

Blanco, Juan

Blank, Miri

Blasiak, Janusz

Blazina, Maria

Blümel, Martina

Bocian, Szymon

Boggia, Raffaella

Bohara, Raghvendra

Böhrnsen, Florian

Bolbasov, Evgeniy

Bölcskei, Hedvig

Bonaccorsi Di Patti, Maria Carmela

Bonaventura, Rosa

Bondon, Arnaud

Bonfili, Laura

Bontempo, Paola

Borges Dos Santos, Rui M.

Borkowska, Monika

Boros, Laszlo

Bortner, Carl D.

Bossowska, Agnieszka

Botana, Ana María

Botelho, Maria Joao

Botta, Bruno

Boudreau, Paul D.

Boukouvalas, John

Boundy, Michael J.

Bourgougnon, Nathalie
Bourguet-Kondracki, Marie Lise

Bowden, Bruce

Bracco, Enrico

Braga, Susana Santos

Bravo, Susana B.

Brecker, Lothar

Brett, Elizabeth

Brown, Alan B.

Brown, Lindsay

Brownlee, Iain A

Bruno, Antonino

Brycki, Bogumil

Bulet, Philippe

Butu, Alina

Cacciola, Francesco

Calado, Cecília R. C.

Calado, Ricardo

Calcinai, Barbara

Caldara, Marina

Calina, Daniela

Cametti, Cesare

Campbell, Christopher

Campera, Marco

Cañada, Francisco

Capillo, Gioele

Capon, Rob

Carballeira, Nèstor $M$.

Carbonell-Barachina, Angel

Carda, Miguel

Cardoza, Rosa Elena

Carmeli, Shmuel

Caruso, Enrico

Caruso, Gabriella

Carvalho, Fatima

Carvalho, Paulo

Casalino, Elisabetta

Casaril, Angela

Casas, François

Casertano, Marcello

Castro, José Javier Fernández

Castro-Muñoz, Roberto

Catani, Martina

Catanzaro, Elena

Cavallaro, Giuseppe

Caviglia, Gian Paolo

Cerra, Bruno

Ćetković, Helena

Chaikuad, Apirat

Chalamaiah, Meram

Chandra-Hioe, Maria Veronica

Chang, Long-Sen 
Chao, Louis Kuoping

Charoensutthivarakul, Sitthivut

Charusanti, Pep

Chatzidoukas, Christos

Chen, Hsin-Yuan

Chen, Jiezhong

Chen, Shuen-Ei

Chenais, Benoit

Chénais, Benoit

Cheng, Chih-Yu

Cheong, Kit Leong

Chernikov, Oleg

Chevrot, Romain

Chianese, Giuseppina

Chiavaroli, Annalisa

Chimienti, Giovanni

Chizhov, Alexander O.

Chlichlia, Katerina

Choi, Changhoon

Choi, Jong-Il

Chyau, Charng-Cherng

Ciccarelli, Renata

Cicero, Arrigo

Cichero, Elena

Ciesielska, Anna

Cieślińska, Anna

Cirrincione, Girolamo

Clair, James La

Clericuzio, Marco

Colavito, Sierra A.

Collier, Jackie

Conlon, Michael

Contaldo, Maria

Coombs, Melanie

Cord-Landwehr, Stefan

Corral, Paulina

Correia-Da-Silva, Marta

Corsaro, Maria Michela

Costa, Pedro M.

Costantino, Valeria

Costa-Pinto, Ana Rita

Cotas, João

Coustau, Christine

Crossman, Lisa

Crüsemann, Max

Cueto, Mercedes

Cui, Qiu

Cutignano, Adele

Cuypers, Bart

Czernel, Grzegorz

D'Agostino, Paul M.
D'Ambra, Isabella

D'Auria, Maria Valeria

Dahiya, Rajiv

Dahong, Li

Damiano, Fabrizio

Damkaci, Fehmi

Danac, Ramona

Daniela, Ailincai

Dansette, Patrick

Darie-Nita, Raluca Nicoleta

Davis, David A.

De Filippis, Barbara

De Francesco, Francesco

De Los Reyes, Carolina

De Lourdes Pereira, Maria

De Mieri, Maria

De Vendittis, Emmanuele

Degl'innocenti, Donatella

Del Pulgar, Sofia Perez

Della Sala, Gerardo

Demchuk, Oleh

Demori, Ilaria

Deslandes, Eric

Desmaële, Didier

Devkota, Hari Prasad

Dewapriya, Pradeep

Di Bari, Lorenzo

Di Cesare Mannelli, Lorenzo

Di Gaetano, Sonia

Di Lorenzo, Francesco

Diacon, Aurel

Dias, Fernanda F. G.

Dingley, Andrew J.

Dinica, Rodica-Mihaela

Dinos, Georgios

Dițu, Lia Mara

Diverdi, Joseph

Dobrzynski, Piotr

Doi, Takayuki

Dolashka, Pavlina

Domalik-Pyzik, Patrycja

Domingues, Rosario

Domínguez, Herminia

Domínguez-Álvarez, Enrique

Domonkos, Ildikó

Dorantes-Aranda, Juan José

Doroftei, Bogdan

Doupnik, Craig A.

Drabowicz, Józef

Du, Deguo

Duan, Delin 
Dufossé, Laurent

Dumic, Jerka

Durmus, Nedim

Duszka, Kalina

Dutartre, Patrick

Dutertre, Sebastien

Dutertre, Sébastien

Dutot, Mélody

Dvoretsky, Alexander G.

Dyshlovoy, Sergey A.

Dzidic, Alen

Dzunkova, Maria

Efremenko, Elena

Einarsson, Hjorleifur

El Mourabit, Haquima

Elinder, Fredrik

Ellinger, Bernhard

El-Sheekh, Mostafa

Engelmann, Peter

Enguita, Francisco J.

Escrig, Nuria Cabedo

Esposito, Roberta

Esteves, Ana Cristina

Ettayapuram-Ramaprasad, Azhagiya Singam

Fabiszewska, Agata

Fakira, Amanda K.

Falcó, Alberto

Farjon, Jonathan

Fedorov, Alexey N.

Felgueiras, Helena

Feng, Sheng-Wei

Fernández-Acero, Francisco Javier

Fernandez-Lafuente, Roberto

Fernando, I. P. Shanura

Fernando, Ilekuttige Priyan Shanura

Ferreira, Antonio

Figadere, Bruno

Figueroa, Félix L.

Figuerola, Blanca

Filip-Psurska, Beata

Finetti, Federica

Fitton, Janet Helen

Florean, Cristina

Flores, Cintia

Flórez-Fernández, Noelia

Fogacci, Federica

Fontana, Angelo

Fontana, Fabrizio

Foubelo, Francisco

Fouillen, Laetitia

Fraga-Corral, Maria
Fruit, Corinne

Fujita, Masaki

Furuita, Hirofumi

Fuwa, Haruhiko

Gabrielli, Maria Gabriella

Găină, Luiza Ioana

Galeazzi, Roberta

Galicka, Anna

Gallo, Monica

Galvagni, Federico

Gama, Sofia

Ganesan, A.

Garbayo, Inés

Garcia, Jose Prieto

Garcia, Ronald

Garcia-Jimenez, Pilar

Gardikis, Konstantinos

Garzoli, Stefania

Gavenonis, Jason

Gebauer, Jan

Gęgotek, Agnieszka

Genovese, Giuseppa

Gentile, Maria Teresa

Gerdol, Marco

Giampietro, Letizia

Giastas, Petros

Giordano, Daniela

Giordano, Guido

Giordano, Paola

Giráldez, Inmaculada

Gisch, Nicolas

Giudice, Angelina Lo

Giuliani, Maria

Giuliano, Michela

Glukhov, Evgenia

Golomazou, Eleni

Gomes, Carlos Martins

Gomes, Joao R.

Gomes, Nelson

Gómez-Pinchetti, Juan Luis

Gomez-Pinilla Cerezo, Maria Natividad

Gomez-Pinilla, Pedro J.

Gonçalves, Ana M. M.

Gonçalves, Micael

Gonkowski, Slawomir

González-Minero, Francisco José

Gonzalez-Vicente, Agustin

Gorbushin, Alexander

Górska, Agata

Górska, Sabina

Gorzelanny, Christian 
Goti, Andrea

Grasselli, Elena

Grimaldi, Annalisa

Grimm, Marcus

Groff, Joseph

Grovel, Olivier

Grzesiak, Jakub

Gubitosa, Jennifer

Guella, Graziano

Guo, Changjun

Guriec, Nathalie

Gustafson, Kirk

Gutierrez, Mar Fernandez

Guzmán, Eduardo

Guzmán-Ruiz, Rocío

Guzow-Krzemińska, Beata

Hachero, Ismael

Hallegraeff, Gustaaf M.

Hamashima, Yoshitaka

Hanas, Jay

Hanif, Novriyandi

Hannan, Md. Abdul

Hansen, Espen

Hantke, Klaus

Haq, Monjurul

Hardy, Rowan S.

Harrison, Paul H. M.

Harvey, Joanne

Harvey, Patricia J.

Harvey, Peta

Hasan, Imtiaj

Hassouna, Amira Ahmed

Haverkamp, Richard G.

Hayashi, Kyoko

Hayashida, Kenji

Hegazy, Mohamed-Elamir F.

Hernández-Daranas, Antonio

Hernandez-Prieto, Miguel A.

Heymann, Dominique

Hicks, Leslie

Higashi, Nobuaki

Hirabayashi, Jun

Hoffman, Angela

Hojan, Katarzyna

Homaeigohar, Shahin

Horakova, Olga

Hotos, George N.

Hou, Chih-Yao

Hrabal, Richard

$\mathrm{Hsu}$, Fong-Fu

Huang, Chun-Yung
Huang, Jinling

Huerta-Yépez, Sara

Hughes, Christopher V.

Hung, Andrew

Hwang, Bang Yeon

Iatridi, Zacharoula

Ibrahim, Mohamed Ali

Ibrahimi, Manar

Imperatore, Concetta

Imperatore, Roberta

Inagaki, Hidetoshi

Inestrosa, Nibaldo C.

Inuzuka, Toshiyasu

Iqbal, Hafiz M. N.

Isaeva, Marina P.

Ishii, Takahiro

Islam, Salman

Itoh, Takafumi

Itoi, Shiro

Iwasaki, Arihiro

Izzo, Irene

Jakas, Andreja

Jang, Kyuyun

Janowski, Miroslaw

Jarończyk, Małgorzata

Jassbi, Amirreza

Jembrek, Maja Jazvinšćak

Jenner, Ronald

Jenssen, Håvard

Jeong, Se Kyoo

Jerković, Igor

Jiang, Guangde

Jimbo, Mitsuru

Jimenez, Carlos

Jiménez, Carlos

Jiménez-Tenorio, Manuel

Jin, Junyan

João, Cotas

João, Silva

Jones, Chris

Jones, Meriel G.

Joo, Hong-Gu

José-Manuel, Leao Martins

Joyce, Susan A.

$\mathrm{Ju}$, Jianhua

Jubeau, Sébastien

Jung, Jee-Hyung

Jurj, Ancuța Maria

Kadokawa, Jun-Ichi

Kafarski, Pawel

Kafarski, Paweł 
Kajihara, Hiroshi

Kalinin, Vladimir

Kalinin, Vladimir I.

Kaminski, Kamil

Kaminski, Tomasz W.

Kamio, Michiya

Kanda, Tatsuo

Kang, Jeong-Hun

Karayannakidis, Panagiotis

Karuppan, Mohan Kumar Muthu

Kasheverov, Igor

Kashman, Yoel

Kasten-Jolly, Jane

Kaszowska, Marta

Katikou, Panagiota

Kato, Nobutaka

Katsuta, Eriko

Katsuyama, Yohei

Kawakami, Yuki

Kawamura, Akira

Kazakova, Oxana B.

Kelly, Claire M.

Kelly, Michelle

Kem, William

Kempken, Frank

Kerch, Garry

Keyzers, Rob

Keyzers, Robert

Khan, Fazlurrahman

Khan, Mohd Sajid

Khotimchenko, Maxim Y.

$\mathrm{Ki}$, Jang-Seu

Kikukawa, Hiroshi

Kim, Chu-Young

Kim, Euikyung

Kim, Hak Jun

Kim, Hyesun

Kim, Jinsik

Kim, Se-Kwon

Kim, Young-Sang

Kimura, Ken-Ichi

Kinnel, Robin

Kishimura, Hideki

Kiuru, Paula

Klar, Agnes

Kleier, Daniel A.

Klettner, Alexa

Klewicka, Elzbieta

KO, Kam Ming

Kobayashi, Daisuke

Kochneva, Galina
Kojima-Yuasa, Akiko

Kokoulin, Maxim S.

Koksharova, Olga A.

Kolesnikova, Sophia A.

Kolobov, Alexandr Alexandrovich

Komaki, Hisayuki

Komba, Shiro

Korolkova, Yuliya

Korzhikova-Vlakh, Evgenia

Kos, Blaženka

Kose, Ayse

Koshino, Hiroyuki

Kostakis, Ioannis

Kostyuk, Vladimir

Kot, Anna Maria

Kotake-Nara, Eiichi

Kotańska, Magdalena

Kotoku, Naoyuki

Kouokam, Joseph Calvin

Koutelidakis, Antonios E.

Koval, Olga

Kozakiewicz, Anna

Kozanecki, Marcin

Kozlov, Sergey

Kozłowska, Joanna

Kozłowska, Justyna

Krokidis, Marios

Król, Ewelina

Królicka, Aleksandra

Krüger, Marcus

Kuenze, Georg

Kulminskaya, Anna A.

Kumar, Anuj

Kuryk, Lukasz

Kuryłowicz, Alina

Kushkevych, Ivan

Kyzas, George

Kyzas, George Z.

Laatsch, Hartmut

Labes, Antje

Ladrat, Christine Delbarre

Laity, Peter R.

Lamas, Alexandre

Lan, Wenjian

Lapaquette, Pierre

Laronze-Cochard, Marie

Lauder, Bob

Laurienzo, Paola

Lauro, Gianluigi

Lauzon, Marc-Antoine

Lavoie, Serge 
Leber, Regina

Lebreton, Jacques

Leclère, Lucas

Lee Chang, Kim Jye

Lee, Chia-Hung

Lee, Dae-Sung

Lee, Hyeyoung

Lee, Hyi-Seung

Lee, I-Ta

Lee, Jun Sik

Lee, Pyeong Chun

Lee, Sang Kook

Lee, Seung-Hong

Lee, Yunkyoung

Lengyel, Imre

Lephart, Edwin D.

Leshchenko, Elena V.

Łęska, Bogusława

Letsiou, Sophia

Lewicki, Sławomir

Lewinska, Anna

Lewis, Richard

Leychenko, Elena

Leyton, Allison

Li, Fuli

Li, Min-Hui

Li, Rongfeng

Li, Zhun

Liang, Po-Huang

Liaw, Chih-Chuang

Lienqueo, María Elena

Lin, Chih-Chien

Lin, Jer-An

Liou, Je-Wen

Littlefield, Bruce A.

Liu, Bin

Liu, Shing-Hwa

Llewellyn, Carole

Loiseau, Nicolas

Loll, Bernhard

Longoni, Silvia Stefania

López, Lucía Méndez

López-López, Manuel

Lorant, Anne

Lordan, Ronan

Lowell, Andrew

Lu, Wen-Chien

Luis, Ana

Luiz, Rodrigo Cabral

Lundholm, Nina

Maceiras, Rocío
Maciej, Strzemski

Madhukar, Burra

Maduro, Morris

Maeda, Hayato

Magarlamov, Timur Yu.

Maggi, Leonard B.

Magriotis, Plato A.

Makarska-Bialokoz, Magdalena

Makhutova, Olesia

Malara, Alessandro

Malfa, Giuseppe Antonio

Malik, Adeel

Mallamaci, Rosanna

Manabe, Yuki

Mancini, Ines

Mándi, Attila

Mangesh, Harish

Mangoni, Alfonso

Manzo, Emiliano

Maramai, Samuele

Marcial-Quino, Jaime

Marcos, Ricardo

Maresca, Marc

Marin, Ricardo Mallavia

Markopoulos, Anastasios

Marquez-Rocha, Facundo J.

Marra, Alberto

Martin, Neil

Martínez, Antonio Rosales

Martínez-Álvarez, Óscar

Martínez-Espinosa, Rosa María

Martín-Gil, Jesus

Martins, Alice

Martins, Rosário

Maruyama, Hiroko

Maskrey, Benjamin

Massiot, Georges

Matos, Ana Rita

Matsuura, Hiroshi

Matte, Alessandro

Mattei, César

Maurer, Stefanie

Máximo, Patrícia

Mccall, Laura-Isobel

Mccarthy, Peter

Mcfadden, Sandra

Mcmahon, Hilary E. M.

Mcphail, Kerry L.

Mediero, Aránzazu

Meghea, Aurelia

Mehariya, Sanjeet 
Mekasha, Sophanit

Mekinić, Ivana Generalić

Melendez-Ortiz, Hector

Menchinskaya, Ekaterina S.

Meslet-Cladiere, Laurence

Mester, Patrick-Julian

Micael, Joana

Michaud-Soret, Isabelle

Milanova, Aneliya

Miller, Ingrid

Millot, Marion

Miloso, Mariarosaria

Milthorpe, Bruce

Minato, Ken-Ichiro

Minkiewicz, Piotr

Minnelli, Cristina

Minto, Robert E.

Miricescu, Daniela

Miserocchi, Giacomo

Mitaine-Offer, Anne-Claire

Mitchell, Miguel

Miyanaga, Akimasa

Miyashita, Kazuo

Mlostoń, Grzegorz

Moeini, Arash

Mogielnicki, Andrzej

Mohammed, Altaf

Moldenhauer, Marcus

Molgó, Jordi

Molinaro, Antonio

Moloney, Mark

Moncalián, Gabriel

Monge, Claire

Monteiro, Pedro

Montero, Olimpio

Morales, Serafin

Moreira, Daniel Carneiro

Morel-Rouhier, Mélanie

Mori, Hirotada

Mori, Tetsushi

Morimura, Shigeru

Morita, Naoki

Morzycki, Jacek W.

Moschovi, Maria

Mot, Augustin

Mouga, Teresa

Mourão, Paulo A. S.

Mozumder, Md. Salatul Islam

Mozzicafreddo, $\mathrm{M}$.

Mroczek, Agnieszka

Mudge, Elizabeth M.
Mukai, Hidehito

Muller, Marc

Mulloy, Barbara

Munekata, Paulo

Muramoto, Koji

Murata, Michio

Murias, Marek

Murkovic, Michael

Murphy, Patrick J.

Muszyński, Siemowit

Myllykallio, Hannu

Nagai, Hiroshi

Nagao, Koji

Nagaoka, Isao

Naghdi, Samira

Nakata, Norio

Nam, Sang-Jip

Namgaladze, Dmitry

Nandin-Erdene, Mandakhbayar

Nanno, Masanobu

Napolitano, Alessandra

Nashmi, Raad

Nathanailides, Kosmas

Navarro, Juan C.

Navarro, Macarena Sánchez

Naviglio, Daniele

Nedialkov, Paraskev T.

Neilson, Andrew

Neumann, Jake

Neureiter, Daniel

Neut, Christel

$\mathrm{Ng}$, Tzi Bun

Nicolau, Elodie

Nishimura, Shinichi

Nitulescu, Mihai

Noble, Peter A.

Nociari, Marcelo

Noda, Masafumi

Norte, Manuel

Norton, Raymond

Novelli, Antonello

Ntaikou, Ioanna

Nugraha, Bramasta

O'Harte, Finbarr

Ochiuz, Lacramioara

Oczkowski, Michał

Odintsova, N. A.

Oh, Chang-Myung

Oh, Dong-Chan

Oh, Hyuncheol

Ohshiro, Takashi 
Ohta, Shinji

Okamura, Yoshiko

Okino, Tatsufumi

Okumura, Ko

Okunade, Adewole L.

Olejnik, Anna

Olichon, Aurélien

Oliveira, Ana

Ong, Eng Shi

Oniszczuk, Anna

Ooi, Chien Wei

Opatz, Till

Oprea, Ovidiu

Opydo-Chanek, Małgorzata

Orefice, Ida

Orfali, Raha

Orlandini, Elisabetta

Ortiz-Sánchez, Elizabeth

Orvalho, Sandra

Oshiro, Naomasa

Otsuki, Takemi

Ovchinnikova, Tatiana V.

Ozaltin, Kadir

Ozeki, Tetsuya

Ozeki, Yasuhiro

Padrão, Jorge

Palenzuela, José Antonio

Palijan, Goran

Pallio, Giovanni

Palumbo, Anna

Palumbo, Carla

Pampalakis, Georgios

Panaro, Maria Antonietta

Papadimitriou, Theodoti

Papini, Emanuele

Park, Ah-Mee

Park, Chulhwan

Park, Jin-Soo

Parsons, Peter

Pasqualetti, Marcella

Passalacqua, Karla

Pateiro, Mirian

Patruno, Antonia

Pavao, Mauro Sergio

Pavic, Aleksandar

Pavlov, Atanas

Pawlak, Aleksandra

Pearce, Cedric

Pearce, Norrie

Pecorelli, Alessandra

Pedersen, Kim B.
Pelin, Marco

Pentak, Danuta

Pepi, Milva

Perdih, Andrej

Pereira, David

Pereira, Florbela

Pereira, José Augusto

Pereira, Leonel

Pereira, Patrícia Alexandra

Pescitelli, Gennaro

Peter, Martin

Pfeiffer, Ilona

Phan, Chin-Soon

Phelix, Clyde F.

Philippopoulos, Athanassios I.

Philips, Neena

$\mathrm{Pi}$, Jiang

Piazza, Stefano

Pickett, Andy

Pickova, Jana

Piecuch, Agata

Piestansky, Juraj

Piggott, Andrew

Pilkington, Lisa

Pina-Perez, Maria Consuelo

Pinheiro, Joaquina

Pinto, Diana Cláudia

Pires, Carla

Pisklak, Dariusz Maciej

Pitucha, Monika

Plastina, Pierluigi

Platta, Harald

Pletzer, Daniel

Pohnert, Georg

Poku, Rosemary A.

Polčic, Peter

Polticelli, Fabio

Pons, Daniel Gabriel

Poquet, Laure

Potaczek, Daniel P.

Poulin, Remington

Pozharitskaya, Olga

Prada, Justina

Pradhan, Bhola Shankar

Priel, Avi

Prieto, Miguel

Prieto, Miguel A.

Prieto, Miguel Ángel

Prim, Damien

Prisic, Sladjana

Procopio, Francesco Andrea 
Pucciarelli, Sandra

Puillandre, Nicolas

Puizina, Jasna

Quaglino, Daniela

Quaglio, Deborah

Quintas, Alexandre

Rabbani, Gulam

Racovita, Stefania

Radulescu, Cristiana

Radwan, Mohamed

Rafael, Robaina

Ragnarsson, Lotten

Rahman, M. Azizur

Rai, Akhilesh

Raja, Rathinam

Ramazzotti, Matteo

Rapeanu, Gabriela

Rateb, Mostafa

Raudonè, Lina

Raugei, Giovanni

Ravi, Rama S.

Ravindran, Rajeev

Rawski, Mateusz

Rebollo-Hernanz, Miguel

Rebuffat, Sylvie

Regina, Giuseppe La

Reis, Cristiano

Reis-Costa, Pedro

Remias, Daniel

Revuelta, Julia

Reyes, Carolina Pérez

Reyes, Fernando

Reynisson, Jóhannes

Rhee, Jae-Sung

Rho, Jung-Rae

Ricciardi, Maria Rosaria

Riccio, Gennaro

Rimondini, Lia

Roberti, Roberta

Rochefort, Gael Y.

Rodrigues, Márcio José

Rodrigues, Maria João

Rodriguez, Isabel

Rodríguez, Jaime

Rodríguez-Yoldi, María Jesús

Roje, Marin

Rok, Jakub

Romagnoli, Romeo

Romano, Giovanna

Rosengren, Johan

Ross, Ian L.
Rossi, Claudio

Roth, Christian

Różalska, Barbara

Rozza, Ariane

Rudolf, Emil

Ruml, Tomas

Ruocco, Nadia

Russell, Fraser

Ryabchikova, Elena I.

Rye, Philip D.

Ryffel, Bernhard

Ryu, Bomi

Sabot, Cyrille

Sadamoto, Hisayo

Sadovskaya, Irina

Saez, Carmen

Saez, Jose Colon

Sáez-Casado, María Isabel

Safavi, Maliheh

Saha, Subhasish

Saini, Ramesh Kumar

Sakai, Ryuichi

Sakayori, Nobuyuki

Šalić, Anita

Salim, Angela

Salinas, Eva

Salomon, Christine

Salvador-Reyes, Lilibeth

Salzano, Anna Maria

Samaja, Michele

Sampedro, Diego

Sanchez-Amat, Antonio

Sanchez-Ruiz, Antonio

Sancho-Vaello, Enea

Sangadala, Sreedhara

Sanjeewa, Kalu Kapuge Asanka

Sansoe, Giovanni

Sansone, Anna

Santabarbara, Stefano

Santamaria, Ramon

Sarabia, Francisco

Saratale, Rijuta Ganesh

Saravanakumar, Kandasamy

Šarkanj, Bojan

Sastre, Leandro

Satake, Masayuki

Satish, Lakkakula

Sato, Shigeru

Saul, Nadine

Saurav, Kumar

Sayed, Ahmed 
Scarfi, Sonia

Schikov, Alexander

Schlein, Christian

Schmid, Jochen

Schrama, Denise

Schroeder, Christina

Scocchi, Marco

Scott, Nicola Jean Agnes

Seale, Lucia A.

Searcey, Mark

Seca, Ana M. L.

Seca, Ana Maria Loureiro

Sedlic, Filip

Seguí Gil, Lucía

Seijas Vázquez, Julio A.

Sela, Shlomo

Selwood, Andy

Semenycheva, Ludmila L.

Seo, Ji-Hun

Serralheiro, Maria Luísa

Seto, Shigeki

Shaala, Lamiaa A.

Sham, Yuk Y.

Sharma, Kavita

Sharma, Nikita

Shavandi, Amin

Shave, Steven

She, Zhi-Gang

Shene, Carolina

Sheu, Jyh-Horng

Shidoji, Yoshihiro

Shigemura, Yasutaka

Shikov, Alexander

Shikov, Alexander N.

Shin, Hee Jae

Shin, Jongheon

Shin-Ya, Kazuo

Shnyder, Steve

Siebert, Hans-Christian

Sil, Susmita

Silachev, Denis

Silchenko, Alexandra S.

Silipo, Alba

Silva, Aurora

Silva, Joana

Silva-Abreu, Marcelle

Silvestre, Samuel

Šindlerová, Lenka

Sing, Swee Leong

Singh, Jonathan

Singla, Bhupesh
Sionov, Edward

Siopa, Filipa

Sipkema, Detmer

Sivanesan, Iyyakkannu

Skorko-Glonek, Joanna

Skouta, Rachid

Skropeta, Danielle

Slamova, Kristyna

Smerilli, Arianna

Smith, G. Jason

Smith, Kerry

Söderhäll, Kenneth

Sogawa, Norio

Solano, Francisco

Solovchenko, Alexei

Song, Fuhang

Song, Myoung Chong

Song, Myoung-Chong

Sorensen, John

Soreq, Hermona

Soriano-Romaní, Laura

Soriano-Ursúa, Marvin A.

Sosa, Silvio

Sosio, Margherita

Sosnowska, Katarzyna

Sousa, Maria João

Sousa, Sérgio

Sovadinova, Iva

Sowa, Ireneusz

Spanò, Nancy

Sperandeo, Paola

Spurio, Roberto

Stanciuc, Ana Maria

Ständker, Ludger

Stefan, Simm

Stefaniuk, Dawid

Stejskal, Vlastimil

Stoskopf, Michael

Stoyneva-Gärtner, Maya

Stringer, Damien N.

Stringham, James M.

Strøm, Morten B.

Strzelecka-Kiliszek, Agnieszka

Stuart, David T.

Studzinska-Sroka, Elzbieta

Sugahara, Takuya

Sugimoto, Sachiko

Sulej, Justyna

Sullivan, Ryan

Sun, Ramon

Surup, Frank 
Suzuki, Iwane

Szaleniec, Maciej

Szekalska, Marta

Szkaradkiewicz, Andrzej

Tabudravu, Jioji N.

Tagliazucchi, Davide

Taguchi, Kensei

Tailhades, Julien

Takaichi, Shinichi

Takashige, Kashimoto

Takashima, Shigeo

Takeshita, Satoshi

Talens-Perales, David

Tam, Roger Y.

Tamarit, Daniel

Tamkovich, Svetlana

Tampucci, Silvia

Tanaka, Junichi

Tanaka, Naonobu

Tang, Haoyu

Tareq, Fakir Shahidullah

Tava, Aldo

Téletchéa, Stéphane

Teodoro, João

Teotia, Arun Kumar

Teragawa, Hiroki

Tersariol, Ivarne L. S.

Teusch, Nicole

Thomas, Eric Jim

Thomas, Olivier

Tilocca, Bruno

Timoshenko, Alexander V.

Tiscar, Pietro G.

Tomás, Juan M.

Tomaszewska, Ewa

Tommonaro, Giuseppina

Tonello, Fiorella

Trček, Janja

Trincone, Antonio

Trofimov, Aleksei V.

Trojanowicz, Bogusz

Trombetta, Maria Federica

Troselj, Koraljka Gall

Tsakovska, Ivanka

Tseng, Chih-Hua

Tsironi, Fanny

Tuvikene, Rando

Tziveleka, Leto-Aikaterini

Ucciferri, Claudio

Ueno, Akio

Ueno, Mikinori
Urban, Philippe

Vafidis, Dimitris

Vaiano, Fabio

Vale, Paulo

Van Wijk, Rob Christiaan

Varna, Mariana

Varvaresou, Athanasia

Vaughan, Mel

Vavitsas, Konstantinos

Vázquez-Tato, M. Pilar

Vázquez-Ucha, Juan C.

Vega-Holm, Margarita

Velázquez-Campoy, Adrian

Veranic, Peter

Vezzi, Alessandro

Vicente, Filipa A.

Vicente, Francisca

Vickery, Karen

Viguera, Ana Rosa

Vijayaanand, Arokia Mariyadoss

Vijayanand, Arokia Mariyadoss

Vilariño, Natalia

Vilchez, Carlos

Vilcinskas, Andreas

Villa, Carla

Vinayak, Vandana

Vinogradov, Evguenii

Vistoli, Giulio

Vitale, Rosa Maria

Vochozka, Marek

Vorob'ev, Mikhail M.

Vostinaru, Oliviu

Wagner, Liane

Wagner, Ludwig

Wang, Bo

Wang, Hui-Min

Wang, San-Lang

Wang, Shumin

Watanabe, Ryuichi

Waugh, Mark G.

Weiss, Étienne

Wempe, Michael

White, Lindsey

Wielgosz-Collin, Gaetane

Willett, Julia

Windshügel, Björn

Wińska, Katarzyna

Wójciak-Kosior, Magdalena

Wojtunik-Kulesza, Karolina A.

Wong, Fung-Fuh

Wöstemeyer, Johannes 
Wu, Chang-Jer

$\mathrm{Wu}$, Cheng-Tien

Yamasaki, Masao

Yamashita, Atsuya

Yan, Ning

Yanagihara, Angel

Yang, Heejung

Yasuyuki, Nogata

Yen, Chia-Hung

Yongsawatdigul, Jirawat

Yoo, Keunje

Yoon, In-Soo

Yoon, Sik

Yotsu-Yamashita, Mari

Yousefi, Morteza

Youssef, Diaa

Yurchenko, Ekaterina

Zabetakis, Ioannis
Zaitsev, Aleksey

Zamyatina, Alla

Zárate, Rafael

Zaravinos, Apostolos

Zastrow, Leonhard

Zeuner, Birgitte

Zhang, Changsheng

Zhang, Fan

Zhang, Junzeng

Zhong, Fangrui

Zhou, Shaobo

Zhu, Yuyang

Zhuravleva, Olesya I.

Zouridakis, Marios

Zubía, Eva

Zugaza, José Luis

Zuk, Magdalena

Zych, Maria 